\title{
Silencing survivin expression inhibits the tumor growth of non-small-cell lung cancer cells in vitro and in vivo
}

\author{
KEJIAN ZHANG, YANG LI, WEI LIU, XINLIANG GAO and KEWEI ZHANG \\ Department of Thoracic Surgery, The First Hospital of Jilin University, Changchun, Jilin 130021, P.R. China
}

Received January 26, 2014; Accepted September 18, 2014

DOI: $10.3892 / \mathrm{mmr} .2014 .2729$

\begin{abstract}
Survivin is a promising anticancer therapeutic target due to its important role in the inhibition of apoptosis of tumor cells. However, little is currently known about its role in non small cell lung cancer (NSCLC). The present study evaluated whether the downregulation of survivin expression would affect cell proliferation, cell cycle distribution, apoptosis and colony formation of NSCLC. A recombinant lentiviral small hairpin RNA (shRNA) expression vector, which specifically targeted survivin, was constructed and transfected into the A549 human NSCLC cell line. Quantitative polymerase chain reaction and western blotting were used to determine the mRNA and protein expression levels of survivin, $48 \mathrm{~h}$ following the knockdown of survivin expression. Cell proliferation, apoptosis, cell cycle distribution and colony formation were determined following the downregulation of survivin by shRNA. In addition, A549 cells were injected into nude mice, and the effects of shRNA targeting the survivin gene on tumor growth were assessed. Downregulation of survivin expression, using the RNA silencing approach in A549 tumor cells, significantly suppressed the proliferation and colony formation ability of the cells, and induced tumor apoptosis in vitro. The nude mice inoculated with A549 cells developed cancer, and treatment with shRNA targeting survivin markedly inhibited the growth of these cancers, with no obvious side effects. The results of the present study suggest that suppression of survivin expression by RNA interference may induce NSCLC apoptosis, and provide a novel approach for anticancer gene therapy.
\end{abstract}

Correspondence to: Dr Kewei Zhang, Department of Thoracic Surgery, The First of Hospital of Jilin University, 71 Xinmin Street, Changchun, Jilin 130021, P.R. China

E-mail: zhangkewei1410@163.com

Key words: non-small-cell lung cancer, survivin, RNA silencing, tumor growth

\section{Introduction}

Non-small-cell lung cancer (NSCLC) accounts for 85\% of all lung cancers (1). NSCLC is the most common cause of cancer-associated mortality worldwide, with $<15 \%$ of patients surviving beyond five years, due to the lack of early diagnosis and effective treatment methods $(2,3)$. Chemotherapy and radiotherapy have been widely used in the treatment of advanced NSCLC; however, the outcome remains unsatisfactory, with low long-term survival rates, therefore a more effective and safer therapy for NSCLC is required (4). With advances in the understanding of the molecular pathways associated with NSCLC progression, targeted therapies that are designed to interfere with cancer cell growth and survival, have potential as novel NSCLC therapeutics.

Survivin, a member of the Inhibitor of Apoptosis Protein (IAP) family (5), has been identified as a promising therapeutic target in cancers, as it has been shown to be overexpressed in a wide range of tumors, including stomach (6), colorectal (7), lung (8), breast (9), pancreatic (10), ovarian (11), prostate cancers (12), and melanoma (13). In addition to its role in the inhibition of apoptosis, survivin has a critical role in the regulation of cell division, by inducing exit from $\mathrm{G}_{1}$ checkpoint arrest and subsequent entry into the S phase (14). Previous studies have demonstrated that survivin expression is low in normal cells, but is high in proliferating cancer cells and during cancer angiogenesis (15). These results suggest that survivin may be a suitable target for anticancer therapy.

Survivin expression is closely associated with the sensitivity of cancer cells to chemotherapy, survival time, and prognosis (16). Inhibition of survivin expression may induce apoptosis, inhibit cancer growth, and enhance the sensitivity of cancer cells to chemotherapy and radiotherapy (17). Previous studies have reported on the transfection of lung cancer cells with small interfering (si)RNA specifically targeting survivin. Chen et al (18) reported that downregulation of survivin expression by RNA interference (RNAi) in A549 cells, markedly decreased the invasive and metastatic capabilities of the cells, suppressed proliferation, decelerated the rate of growth, reduced the number of clones on soft agar and decreased the capacity of reconstituted basement membrane penetration and migration. However, this study did not investigate the responses in an in vivo model.

In the present study, the effects of RNAi targeting survivin were examined, on cell proliferation, cell cycle 
distribution and cell apoptosis in the A549 human NSCLC cell line in vitro and on tumor growth in a lung cancer xenograft in vivo.

\section{Materials and methods}

Cell culture. The A549 human NSCLC and HEK 293 T human embryonic kidney cells were purchased from the Cell Bank of Type Culture Collection of Chinese Academy of Sciences, Shanghai Institute of Cell Biology, Chinese Academy of Sciences (Shanghai, China). The A549 and HEK 293T cells were cultured in RPMI-1640 medium (Invitrogen Life Technologies, Carlsbad, CA, USA), supplemented with heat-inactivated $10 \%$ fetal bovine serum (FBS) (Biochrom $\mathrm{AG}$, Berlin, Germany) at $37^{\circ} \mathrm{C}$, in a humidified atmosphere containing $5 \% \mathrm{CO}_{2}$.

Construction of survivin small hairpin (sh)RNA-containing lentivirus and infection. The pshRNA-H1-Luc lentivector (System Biosciences, Mountain View, CA, USA) used in the present study was designed to coexpress luciferase, cloned from the Copepod. The shRNA sequence targeting the human survivin gene was as follows: Sense, 5'-GGCTGGCTTCATCCACTGC-3', and has previously been shown to efficiently downregulate human survivin expression (18). The shRNA sequence used for the scrambled negative control (NC) was: Sense, 5'-AATTCTCCGAACGTGTCACGT-3'. This sequence did not target any known gene product and had no significant sequence homology with human gene sequences, which is essential for determining the effects of shRNA delivery. Pairs of complementary oligonucleotides containing these sequences were synthesized (Invitrogen Life Technolgies) and cloned into the pshRNA-H1-Luc lentivector. The pshRNA-H1-Luc lentivectors containing the shRNA sequences and the pPACK Packaging Plasmid Mix (System Biosciences, Shanghai, China) were cotransfected into HEK 293T producer cells using Lipofectamine ${ }^{\circledR} 2000$ (Invitrogen Life Technologies). The viral supernatants were harvested following $48 \mathrm{~h}$, and the titers were determined with serial dilutions of concentrated lentivirus.

Quantitative polymerase chain reaction ( $q P C R$ ). The A549 and HEK 293T cells were harvested for RNA extraction $48 \mathrm{~h}$ after shRNA treatment. The RNA was extracted using the RNeasy Mini kit, with the RNase-free DNase Set to remove any contaminating genomic DNA (Qiagen, Valencia, CA, USA). The RNA was reverse-transcribed into cDNA using the Primescript ${ }^{\mathrm{TM}}$ RT reagent kit, according to the manufacturer's instructions (Takara Bio Inc., Otsu, Japan). The qPCR assays were performed using the $\mathrm{SYBR}^{\circledR}$ Green Real-Time PCR Master Mix (Toyobo Co., Ltd., Osaka, Japan) and qPCR amplification equipment (ABI 7900 Fast System; Applied Biosystems Life Technologies, Foster City, CA, USA) using the following specific primers: Survivin forward, 5'-AGAACTGGCCCTTCTTGGAGG-3', and reverse, 5'-CTTTTTATGTTCCTCTATGGGGTC-3'; GAPDH forward, 5'-TGTGGGCATCAATGGATTTGG-3', and reverse, 5'-ACACCATGTATTCCGGGTCAAT-3'. The PCR conditions were used as follows: Pre-denaturing at $95^{\circ} \mathrm{C}$ for $5 \mathrm{~min}$, followed by 40 cycles of denaturation at $95^{\circ} \mathrm{C}$ for $10 \mathrm{sec}$, annealing/extension at $60^{\circ} \mathrm{C}$ for $15 \mathrm{sec}$, and a final extension at $72^{\circ} \mathrm{C}$ for $10 \mathrm{~min}$. The specificity of the amplification was checked using a melting curve analysis. The expression of the genes of interest were determined by normalization of the cycle threshold $(\mathrm{Ct})$ of the target genes to that of the control GAPDH. The $2^{-\triangle \Delta C T}$ method was used to calculate the relative abundance of the target gene expression levels, generated by Rotor-Gene 6000 Series Software 1.7 (Qiagen, Hilden, Germany). Each sample was performed in triplicate.

Western blotting. The cells were lysed in $100 \mu 1$ radioimmunoprecipitation assay buffer. The proteins were then quantified using the bicinchoninic acid kit (Pierce Biotrchnology Inc., Rockford, IL, USA). A total of $50 \mu \mathrm{g}$ of protein samples were loaded and separated by $8-15 \%$ SDS-PAGE and transferred onto nitrocellulose membranes (Santa Cruz Biotechnology, Inc., Dallas, TX, USA). The membranes were blocked in tris-buffered saline (TBS), containing 5\% skim milk and $0.1 \%$ Tween ${ }^{\circledR} 20$ for $2 \mathrm{~h}$ at room temperature, and then incubated with the following antibodies: Mouse anti-Caspase-3 monoclonal antibody (1:500 dilution), mouse anti-Caspase- 8 monoclonal antibody (1:500 dilution), rabbit anti-survivin polyclonal antibody (1:1,000 dilution; Cell Signaling Technologies, Danvers, MA, USA) and mouse anti-GAPDH monoclonal antibody (1:1,000 dilution; Ambion Life Technologies, Carlsbad, CA, USA) for $2 \mathrm{~h}$. The membranes were then incubated with anti-rabbit or anti-mouse secondary horseradish peroxidase conjugated antibodies (1:10,000 dilutions; GE Healthcare Life Sciences, Uppsala, Sweden) for $2 \mathrm{~h}$. The protein bands were visualized using an Enhanced Chemiluminescence reagent (GE Healthcare, Vélizy-Villacoublay, France).

Measurement of A549 cell viability by MTT assay. To measure the effects of downregulation of survivin expression by shRNA, on cell proliferation, an MTT assay was performed. A total of $5 \times 10^{4} \mathrm{~A} 549$ cells $/ \mathrm{ml}$ were added to a 96-well plate (100 $\mu \mathrm{l} /$ well). In the blank control wells, $100 \mu \mathrm{l}$ of medium alone was added. Following a $24 \mathrm{~h}$ culture, the cells were transfected with a lentivirus expressing an shRNA targeting survivin. The cells were divided into three groups: Normal control, negative control (NC), and survivin shRNA groups. There were eight wells of cells cultured for each group. Following a $48 \mathrm{~h}$ culture, $20 \mu \mathrm{l}$ MTT $(5 \mathrm{mg} / \mathrm{ml})$ was added to each well, followed by an incubation at $37^{\circ} \mathrm{C}$ for $48 \mathrm{~h}$. The plates were then centrifuged at $2,000 \mathrm{x}$ g for $10 \mathrm{~min}$. The supernatants were removed, and $200 \mu 1$ dimethyl sufoxide was added to each well, followed by agitation for $10 \mathrm{~min}$. The absorbance was measured at $570 \mathrm{~nm}$ using a microplate reader (Molecular Devices, Sunnyvale, CA, USA), and the inhibition of growth was calculated. The mean proliferation of cells without any treatment was expressed as $100 \%$.

Cell colony formation assay. The cell suspensions containing shRNA $\left(1 \times 10^{4}\right.$ diluted in $0.33 \%$ low-melting agarose) were overlaid on a bottom $0.5 \%$ agar layer $(3 \mathrm{ml})$ in a $60-\mathrm{mm}$ dish. The cells were incubated at $37^{\circ} \mathrm{C}$ for 14 days, and the medium was refreshed every three days. Following the incubation, 
the colonies were washed twice with phosphate-buffered saline (PBS), fixed with ice-cold methanol for $30 \mathrm{~min}$ and stained with giemsa (Dingguo Biotechnology, Co., Ltd., Beijing, China) for $10 \mathrm{~min}$. The visible colonies were counted.

Cell cycle analysis. To determine the cell cycle distribution, $5 \times 10^{5}$ A549 cells were plated in 60-mm dishes and treated with NC or survivin shRNA for $48 \mathrm{~h}$. Following treatment, the cells were collected by trypsinization, fixed in $70 \%$ ethanol, and maintained at $-20^{\circ} \mathrm{C}$ overnight for fixation. The cells were then washed in PBS, resuspended in $1 \mathrm{ml}$ of PBS containing $100 \mu \mathrm{g} / \mathrm{ml}$ RNase and $40 \mu \mathrm{g} / \mathrm{ml}$ propidium iodide (PI) (Merck, Whitehouse Station, NJ, USA) and incubated in the dark for $30 \mathrm{~min}$ at room temperature. The distribution of the cells in the cell cycle phases were analyzed from the DNA histogram using a FACSCaliber ${ }^{\mathrm{TM}}$ flow cytometer and CellQuest $^{\text {TM }}$ software (BD Biosciences, San Jose, CA, USA).

Detection of apoptosis. The A549 cells were cultured in six-well plates in RPMI-1640, containing 10\% FBS medium, and were treated with NC or survivin shRNA for $48 \mathrm{~h}$. The cover slips were washed three times with PBS $(\mathrm{pH}=7.2)$ and single cell suspensions were fixed in $1 \%$ PBS. The cells were stained with $100 \mu \mathrm{g} / \mathrm{ml}$ acridine orange and $100 \mu \mathrm{g} / \mathrm{ml}$ ethidium bromide for $1 \mathrm{~min}$. The cells were observed under a fluorescence microscope (Olympus Corporation, Tokyo, Japan). A minimum number of 200 cells were counted and the percentage of apoptotic cells was determined. At the molecular level, caspase- 3 and caspase- 8 protein expression levels were detected by western blotting, which provided an additional indicator of apoptosis.

Antitumor effects of survivin shRNA in vivo. All animal experiments were performed in accordance with institutional guidelines, following a protocol approved by the Ethics Committees of the Disease Model Research Center, the First Hospital of Jilin University (Changchun, China). Female BALB mice, $\sim 6-7$ weeks old, were purchased from the Institute of Laboratory Animal Science, Jilin University (Changchun, China) and maintained under specific pathogen-free conditions and provided with food and water ad libitum. One week prior to the experiment the mice were fed with a normal pellet diet.

The A549 cells in the exponential growth phase were harvested and single-cell suspensions $\left(2 \times 10^{6}\right.$ cells $/ 100 \mu \mathrm{l}$ PBS) were injected subcutaneously into the right dorsal flank of the mice. The size of the tumors were measured every 2-3 days, and the tumor volume was calculated as $0.5236 \mathrm{x}$ width ${ }^{2} \mathrm{x}$ length. When the tumors had grown to an average volume of $75 \mathrm{~mm}^{3}$, the mice were randomly divided into survivin shRNA, NC and control groups ( $\mathrm{n}=10 /$ group) and treated with survivin shRNA or NC shRNA, plus PBS, in a total volume of $20 \mu \mathrm{l}$ (10 $\mu \mathrm{l}$ virus plus $10 \mu \mathrm{l}$ PBS) once a week for 21 days. When the control mice began to succumb to the tumors, the mice in all treatment groups were sacrificed. After the mice had been sacrificed, the tumors were removed and directly embedded in an optimal cutting temperature compound and frozen at $-80^{\circ} \mathrm{C}$.

Statistical analysis. All data are expressed as the means \pm standard deviation. The statistical analyses between two samples was performed using a student's t test. A statistical comparison of more than two groups was performed using a one-way analysis of variance, followed by a Tukey post hoc test. Graphpad Prism 5.0 software (GraphPad Software, San Diego, CA, USA) was used for all statistical analyses. A $\mathrm{P}<0.05$ was considered to indicate a statistically significant difference.

\section{Results}

Survivin gene silencing efficiency in A549 NSCLC cells. Previously validated survivin shRNA sequences (18), were cloned into the pshRNA-H1-Luc lentivector and transfected into HEK 293T cells. The silencing efficiency of the lentiviruses was determined at both the mRNA and protein expression levels in the A549 cells. A shRNA sequence presenting no homology with mRNA databases, was used as a negative control. A qPCR and western blot analyses were performed to detect the mRNA and protein expression levels of survivin, three days post-transfection. There was no significant inhibition of survivin mRNA expression levels in the NC and control groups. However, the mRNA expression levels of survivin in the survivin shRNA group were significantly downregulated, as compared with the $\mathrm{NC}$ and control groups (Fig. 1A, $\mathrm{P}<0.01$ ).

There was also no significant inhibition in the protein expression levels of survivin in the NC and control groups, whereas the band density was markedly decreased in the survivin shRNA group, as compared with the NC and control groups (Fig. 1B, $\mathrm{P}<0.01$ ). These results suggest that silencing survivin expression with shRNA significantly decreased the expression levels of survivin in NSCLC cells.

Effects of survivin shRNA on cell proliferation and colony formation. Following transfection of the cells with survivin shRNA, the effects of silencing survivin expression were determined on tumor cell growth in vitro. The anti-proliferative effects of survivin expression silencing on A549 tumor cells were examined using an MTT assay.

Transfection of A549 cells with shRNA targeting survivin inhibited cell proliferation, as compared with the control and NC groups (Fig. 2A, $\mathrm{P}<0.01$ ). The effects of silencing survivin expression on NSCLC cell colony formation ability were also assessed. Silencing survivin expression reduced the number of tumor cell colonies, as compared with the $\mathrm{NC}$ and control groups (Fig. 2B; $\mathrm{P}<0.01$ ).

Effects of survivin shRNA on cell apoptosis and cell cycle distribution. To investigate whether silencing survivin expression may induce apoptosis, the percentage of apoptotic cells was determined following treatment with shRNA targeting survivin. The A549 cells treated with survivin shRNA had a significantly higher rate of apoptosis, as compared with the control and $\mathrm{NC}$ groups (Fig. 3A; $\mathrm{P}<0.01$ ).

Flow cytometry was performed to assess the effects of survivin shRNA on the progression of the cell cycle in A549 cells. A comparison of the cell cycle distribution of the survivin shRNA group with the control and NC groups, indicated that the survivin shRNA group had a significantly higher proportion of cells in $G_{1}$ and $G_{2}$ phases, and fewer 
A

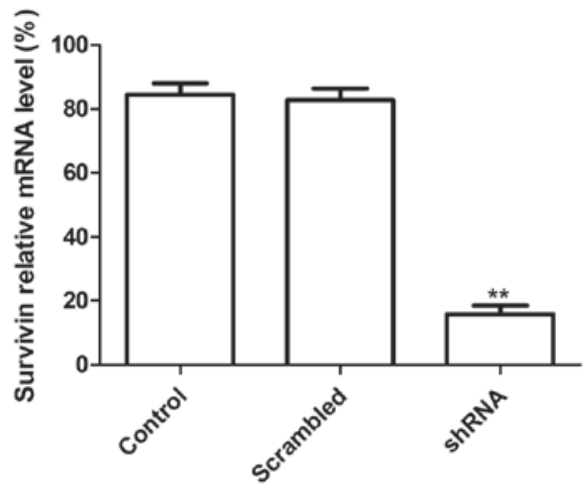

B

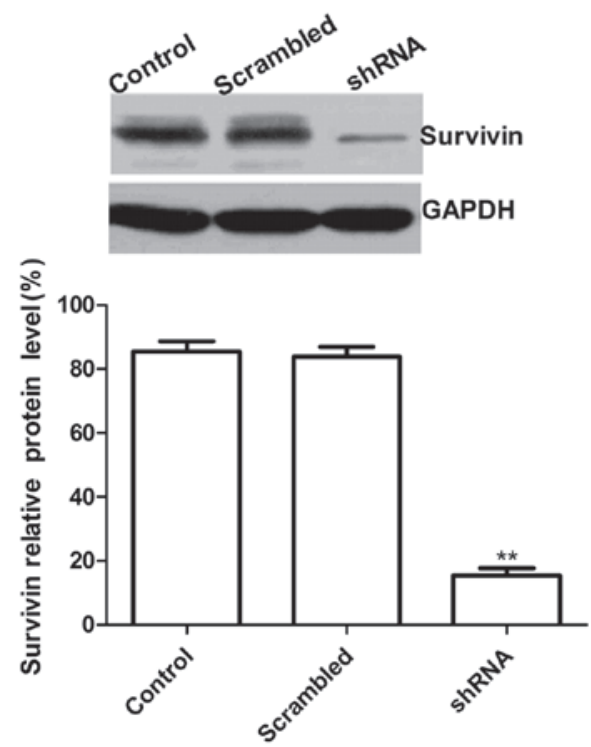

Figure 1. Silencing survivin expression inhibited survivin expression in A549 non-small-cell lung cancer tumor cells. (A) Quantitative polymerase chain reaction and (B) western blotting analysis of survivin, following small hairpin (sh)RNA. The data represent the means \pm standard deviation. ${ }^{* *} \mathrm{P}<0.01 \mathrm{vs}$ control.

A

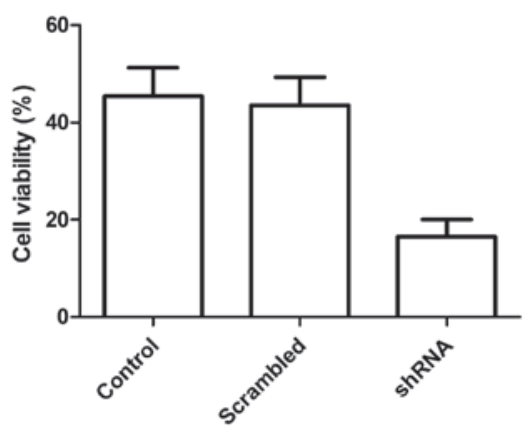

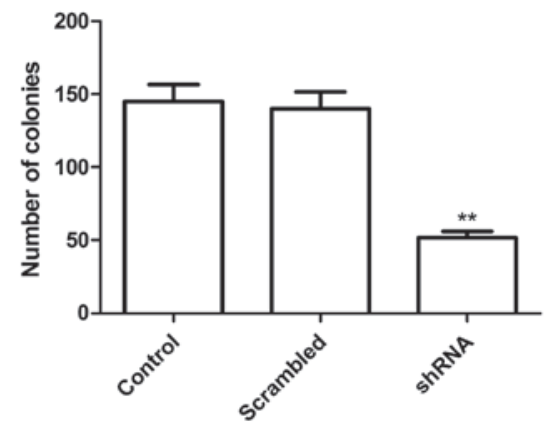

Figure 2. Silencing survivin expression inhibited cell proliferation and cell colony forming abilities in vitro. Downregulation of survivin by transfection of small hairpin (sh)RNA significantly suppressed the (A) proliferation, and (B) cell colony formation of A549 human non-small-cell lung cacncer cells. The data represent the means \pm standard deviation. ${ }^{* *} \mathrm{P}<0.01$ vs control.

cells in $\mathrm{S}$ phase (Fig. 3B; $\mathrm{P}<0.05$ ). There were no significant differences between the control and the $\mathrm{NC}$ groups.

Effects of survivin shRNA on caspase-3 and caspase- 8 expression. To determine the effects on apoptosis following silencing survivin expression, the protein expression levels of caspase-3 and caspase- 8 were analyzed by western blotting. Silencing survivin expression markedly increased the expression levels of caspase-3 and caspase-8, in A549 cells, as compared with the control and $\mathrm{NC}$ groups (Fig. 4).

Survivin shRNA slows tumor growth in a murine xenograft model. The effects of survivin shRNA were also investigated on tumor growth in mice with lung cancer xenografts. There were no significant differences in tumor volume pre-treatment, and the mice from all three groups had clearly evident tumors at 21 days. The tumor volume was significantly reduced in the survivin shRNA group on days 7, 14 and 21 (Fig. 5A; $\mathrm{P}<0.001$ for all).

Three days after the end of the treatment, the mice were sacrificed, and the weight of the tumors were measured. The tumor weight was significantly less in the survivin shRNA group, as compared with the control and $\mathrm{NC}$ groups (Fig. 5B; $\mathrm{P}<0.001)$. These results demonstrate that silencing survivin expression may suppress tumor growth of NSCLC in vivo.

\section{Discussion}

RNAi is a promising approach for antitumor therapy that allows in vivo silencing of essential genes for tumor progression. RNAi provides an alternative to traditional small molecule therapies, and has generated a great deal of interest in treating cancer. However, success of this treatment method depends on the identification of a gene which is expressed universally in cancer cells, but not in normal cells. Survivin may be a potential candidate molecule to target for antitumor therapy. Extensive studies have shown that survivin is upregulated in various human cancers, including lung cancer (6-13), but not in normal prostatic tissues. In addition, a previous study showed that elevated survivin expression is associated with chemoresistance (19-21). Therefore, targeted suppression of survivin expression may be a potential 
A

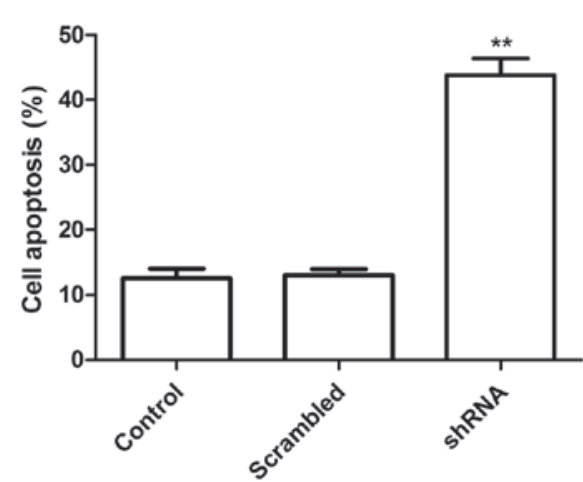

B

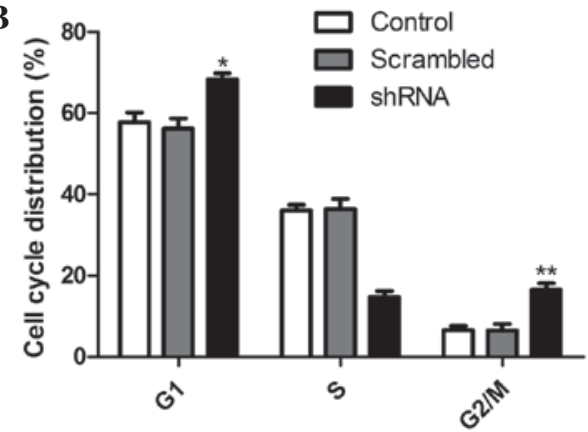

Figure 3. Effects of silencing survivin expression on apoptosis and cell cycle distribution in vitro. Downregulation of survivin by transfection of small hairpin (sh)RNA (A) induced apoptosis and (B) suppressed the cell cycle of A549 human non-small-cell lung cancer cells. The data represent the means \pm standard deviation. ${ }^{*} \mathrm{P}<0.05,{ }^{* *} \mathrm{P}<0.01$ vs control.

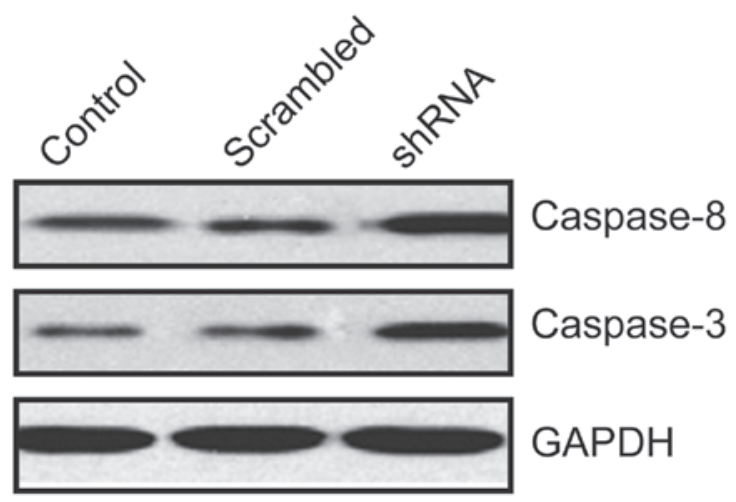

Figure 4. Silencing survivin expression affects the expression levels of apoptosis-associated proteins, as determined by western blotting. The protein expression levels of caspase-3 and caspase-8 were increased following downregulation of survivin by transfection with small hairpin (sh)RNA targeting survivin.

A

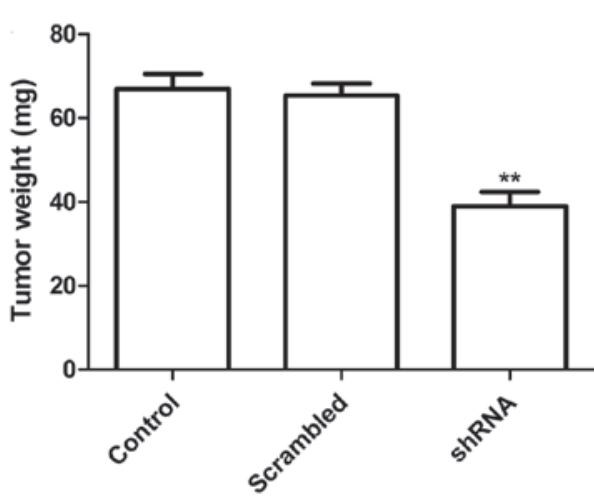

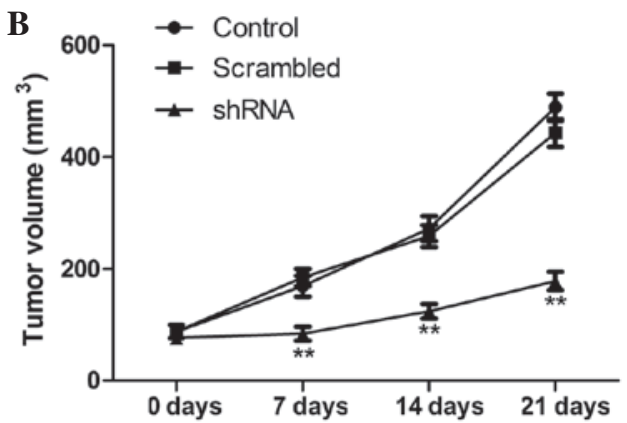

Figure 5. Silencing survivin expression significantly suppressed tumor growth in vivo. (A) Tumor weight in xenograft mice 21 days following silencing by small hairpin (sh)RNA targeting survivin and (B) tumor volume curves of A549 cells following survivn shRNA. The data represent the means \pm standard deviation. ${ }^{* * *} \mathrm{P}<0.01$ vs control.

therapeutic strategy for lung cancer. In the present study, silencing survivin expression in A549 NSCLC cells using a specific shRNA resulted in a decreased cell growth and increased apoptotic rate, these findings are consistent with previous reports (18).

Survivin, a member of the IAP family, has previously been reported to be associated with the cell cycle (15). Numerous studies have shown that survivin protein is synthesized and expressed at high levels during the $\mathrm{G}_{2} / \mathrm{M}$ phase of the cancer cell division cycle, effectively supporting the active cell growth process (22-25); therefore, inhibition of survivin may preferentially block cell division. The present study showed that silencing survivin expression resulted in a significantly higher proportion of cells in $\mathrm{G}_{1}$ and $\mathrm{G}_{2}$ phases fewer cells in $\mathrm{S}$ phase, and more apoptotic cells, which may help to block cell division.

Previous studies have shown that an increased expression of survivin in cancer tissues is associated with a short survival 
time and poor prognosis. Silencing of survivin expression has been demonstrated to induce apoptosis, inhibit the growth of cancer cells, and enhance the sensitivity of cancer cells to chemotherapy and/or radiotherapy $(11,18,26)$. These findings suggest that survivin may be a potential target for the diagnosis and treatment of various types of cancer. The results of the present study showed that downregulation of survivin expression, using an RNA silencing approach, in A549 NSCLC cells significantly suppressed the proliferation and colony forming ability of the cells, and induced tumor apoptosis in vitro, and suppressed tumor growth of NSCLC in vivo. These results further confirm that inhibition of survivin expression may suppress tumor growth.

In conclusion, the present study demonstrated that silencing survivin expression may significantly suppress the proliferation and cell cycle distribution in vitro, and tumor growth in vivo of NSCLC. These results suggest that shRNA targeting survivin may have therapeutic potential for the treatment of NSCLC.

\section{References}

1. Jemal A, Siegel R, Ward E, et al: Cancer statistics, 2009 CA Cancer J Clin 59: 225-249, 2009.

2. Jemal A, Siegel R, Xu J and Ward E: Cancer statistics, 2010. CA Cancer J Clin 60: 277-300, 2010.

3. Reungwetwattana T, Weroha SJ and Molina JR: Oncogenic pathways, molecularly targeted therapies, and highlighted clinical trials in non-small-cell lung cancer (NSCLC). Clin Lung Cancer 13: 252-266, 2012.

4. Roy M, Luo YH, Ye M and Liu J: Nonsmall cell lung cancer therapy: insight into multitargeted small-molecule growth factor receptor inhibitors. Biomed Res Int 2013: 964743, 2013.

5. Ambrosini G, Adida C and Altieri DC: A novel anti-apoptosis gene, survivin, expressed in cancer and lymphoma. Nat Med 3: 917-921, 1997.

6. Lu CD, Altieri DC and Tanigawa N: Expression of a novel antiapoptosis gene, survivin, correlated with tumor cell apoptosis and p53 accumulation in gastric carcinomas. Cancer Res 58: 1808-1812, 1998

7. Kawasaki H, Altieri DC, Lu CD, et al: Inhibition of apoptosis by survivin predicts shorter survival rates in colorectal cancer. Cancer Res 58: 5071-5074, 1998.

8. Monzó M, Rosell R, Felip E, et al: A novel anti-apoptosis gene: Re-expression of survivin messenger RNA as a prognosis marker in non-small-cell lung cancers. J Clin Oncol 17: 2100-2104, 1999.
9. Tanaka K, Iwamoto S, Gon G, et al: Expression of survivin and its relationship to loss of apoptosis in breast carcinomas. Clin Cancer Res 6: 127-134, 2000.

10. Liggins C, Orlicky DJ, Bloomquist LA and Gianani R: Developmentally regulated expression of survivin in human pancreatic islets. Pediatr Dev Pathol 6: 392-397, 2003.

11. Xing J, Jia CR, Wang Y, Guo J and Cai Y: Effect of shRNA targeting survivin on ovarian cancer. J Cancer Res Clin Oncol 138: 1221-1229, 2012.

12. Krajewska M, Krajewski S, Banares S, et al: Elevated expression of inhibitor of apoptosis proteins in prostate cancer. Clin Cancer Res 9: 4914-4925, 2003.

13. McKenzie JA and Grossman D: Role of the apoptotic and mitotic regulator survivin in melanoma. Anticancer Res 32: 397-404, 2012.

14. Wheatley SP and McNeish IA: Survivin: a protein with dual roles in mitosis and apoptosis. Int Rev Cytol 247: 35-88, 2005.

15. Altieri DC: Survivin and IAP proteins in cell-death mechanisms. Biochem J 430: 199-205, 2010.

16. Xing J, Jia CR, Wang Y, Guo J and Cai Y: Effect of shRNA targeting survivin on ovarian cancer. J Cancer Res Clin Oncol 138: 1221-1229, 2012.

17. Zhen HN, Li LW, Zhang W, et al: Short hairpin RNA targeting survivin inhibits growth and angiogenesis of glioma U251 cells. Int J Oncol 31: 1111-1117, 2007.

18. Chen XQ, Yang S, Kang MQ, et al: Survivin expression in human lung cancer and the influence of its downregulation on the biological behavior of human lung cancer cells. Exp Ther Med 3: 1010-1014, 2012.

19. Virrey JJ, Guan S, Li W, et al: Increased survivin expression confers chemoresistance to tumor-associated endothelial cells. Am J Pathol 173: 575-585, 2008.

20. Zhang M, Mukherjee N,Bermudez RS, et al: Adenovirus-mediated inhibition of survivin expression sensitizes human prostate cancer cells to paclitaxel in vitro and in vivo. Prostate 64: 293-302, 2005.

21. Yang $\mathrm{H}, \mathrm{Fu} \mathrm{JH}, \mathrm{Hu} \mathrm{Y}$, et al: Influence of siRNA targeting survivin on chemosensitivity of H460/cDDP lung cancer cells. J Int Med Res 36: 734-747, 2008.

22. Yamazaki H, Takagi S, Hoshino Y, Hosoya K and Okumura M: Inhibition of survivin influences the biological activities of canine histiocytic sarcoma cell lines. PLoS One 8: e79810, 2013.

23. Yamanaka K, Nakahara T, Yamauchi T, et al: Antitumor activity of YM155, a selective small-molecule survivin suppressant, alone and in combination with docetaxel in human malignant melanoma models. Clin Cancer Res 17: 5423-5431, 2011.

24. Chen J, Pise-Masison CA, Shih JH, et al: Markedly additive antitumor activity with the combination of a selective survivin suppressant YM155 and alemtuzumab in adult T-cell leukemia. Blood 121: 2029-2037, 2013.

25. Asanuma K, Tsuji N, Endoh T, Yagihashi A and Watanabe N: Survivin enhances Fas ligand expression via up-regulation of specificity protein 1-mediated gene transcription in colon cancer cells. J Immunol 172: 3922-3929, 2004.

26. Yamamoto H, Ngan CY and Monden M: Cancer cells survive with survivin. Cancer Sci 99: 1709-1714, 2008. 